\title{
Recomendação de conteúdo personalizada com base em estilos de aprendizagem: uma abordagem prática
}

Personalized content recommendation based on learning styles: a practical approach

\author{
Daniel Teixeira Resende \\ Universidade Federal de Uberlândia \\ Faculdade de Computação - FACOM \\ Campus Santa Mônica - Bloco 1B - Sala 1B148 \\ Av. João Naves de Ávila, 2.121 - Bairro Santa Mônica \\ CEP 38400-902 - Uberlândia/MG \\ danieltgr@hotmail.com
}

\author{
Fabiano Azevedo Dorça \\ Universidade Federal de Uberlândia \\ Faculdade de Computação - FACOM \\ Campus Santa Mônica - Bloco 1B - Sala 1B148 \\ Av. João Naves de Ávila, 2.121 - Bairro Santa Mônica \\ CEP 38400-902 - Uberlândia/MG \\ fabiano@facom.ufu.br
}

\begin{abstract}
Resumo A adaptação de fornecimento de conteúdo em sistemas voltados para o ensino é uma área de pesquisa em franca expansão. Isto é explicado por trabalhos que demonstram que estudantes tendem a ter um maior aproveitamento quando a apresentação do conteúdo é personalizada. Neste contexto, os estilos de aprendizagem dos estudantes devem ser observados, sendo esta uma das mais importantes características a serem consideradas no processo de adaptatividade nestes sistemas. Desta forma, este trabalho apresenta uma abordagem eficiente para personalização do processo de ensino, que se baseia no mapeamento automático de características de estilos de aprendizagem de estudantes em metadados de objetos de aprendizagem. Resultados promissores foram obtidos através de experimentos, e são apresentados neste trabalho.
\end{abstract}

Palavras-chave: recomendação personalizada, modelagem do estudante, estilos de aprendizagem, objetos de aprendizagem.

\begin{abstract}
Content adaptation in educational systems is a research area in full expansion. This is explained by studies showing that students tend to have better performances when the content delivery is customized. In this context, student's learning styles should be considered, due to the importance of this feature to the adaptivity process in such systems. Thus, this work presents an efficient approach for personalization of the teaching process based on learning styles. Our approach is based on the automatic mapping of student's learning styles into learning objects metadata. Promising results have been obtained through experiments, which are presented in this paper.
\end{abstract}

Keywords: personalized recommendation, student modeling, learning styles, learning objects. 


\section{Introdução}

Segundo [27], a evolução da tecnologia permitiu a professores e estudantes a utilização do computador como recurso de ensino. Atualmente é possível que os professores, mesmo sem conhecimentos avançados em informática, organizem roteiros de aulas digitais. Porém, os ambientes clássicos de Educação a Distância (EaD), em que prevalecem o modelo de ensino tradicional (um professor, vários estudantes) de maneira convencional (um mesmo conteúdo apresentado igualmente a todos estudantes), possuem, segundo [2], diversas limitações. Pode-se apontar a falta de controle de avaliação personalizada e de adaptação de conteúdo às características dos estudantes. Desta forma, na maioria dos ambientes de $\mathrm{EaD}$ existentes, a interatividade desejável ainda não se encontra disponível [6].

Ao se observar o contexto atual, pode-se perceber que a maioria das instituições de ensino utilizam os chamados Sistemas de Gerenciamento de Aprendizagem ( $\mathrm{Le}$ arning Management Systems - LMS), como Moodle, BlackBoard e Sakai. Estes são desenvolvidos para dar suporte a professores criarem, administrarem e manterem cursos online, provendo uma grande variedade de recursos de ensino e atividades que podem ser facilmente incluídos em cursos. Entretanto, esses sistemas fornecem exatamente o mesmo curso para todos os estudantes e carecem de apoio para professores realizarem adaptações, o que pode ser uma das razões pelas quais os sistemas adaptativos são raramente usados em instituições de ensino [15].

Além da ausência de uma assistência personalizada e inteligente, outro aspecto a ser observado é que estudantes neste tipo de curso podem apresentar perfis bastante diferenciados [6]. Desta forma, com base nos trabalhos de [16] e [25] percebe-se que a adaptação de conteúdo pode prover benefícios a esta modalidade de ensino.

Por isto, é interessante que se possa considerar as particularidades de cada indivíduo, que possui suas próprias preferências em relação à aprendizagem e, com isso, tende a ter um maior aproveitamento à medida que a apresentação do conteúdo a ser estudado esteja mais direcionada ao seu perfil. Nesse contexto, surgem abordagens de personalização de ensino e adaptação de conteúdo de acordo com as características do estudante [1] [24].

Por consequência, a personalização em ambientes de ensino de acordo com as preferências do estudante tem sido um tema amplamente estudado. O principal objetivo dessa personalização é fornecer ao estudante serviços e informações que sejam relevantes ao processo do ensinoaprendizagem e que ao mesmo tempo atendam ao seu perfil [40].

Além disso, os avanços tecnológicos propiciaram avanços no estudo na área de Informática na Educação. A incorporação do termo Objetos de Aprendizagem (OA), definido pelo órgão IEEE [18], e seu padrão de metadados para Objetos de Aprendizagem (LOM - Learning Object Metadata), contribuiu para este avanço. Os OA são entidades - digitais ou não digitais - que podem ser usadas e reutilizadas para ensino, educação ou treinamento.

No que tange às teorias educacionais, Felder e Silverman [11] desenvolveram um modelo das características de Estilos de Aprendizagem (EA) de estudantes por meio de quatro dimensões em que cada uma possui dois polos. A preferência de um estudante pode ser considerada balanceada para uma dimensão, caso não exista tendência clara a um estilo. Também, pode ser considerada moderada ou forte, caso exista uma tendência clara de preferência para um ou outro EA da dimensão. O objetivo da pesquisa era comparar os perfis mais comuns entre os estudantes e o modo de ensino dos professores. As dimensões e os EA inseridos em cada dimensão são apresentados no próximo capítulo.

A inviabilidade de se obter, manualmente, os OA mais adequados aos EA do estudante, em qualquer que seja o repositório, é fundamentada no fato de que há uma grande variação de combinações de características de EA de estudantes neste tipo de curso. Essa circunstância pode ser ainda mais contundente quando se considera repositórios que apresentam número elevado de objetos a serem analisados. É nesse contexto que surge a necessidade por um mecanismo eficaz que realize a correlação automática entre os EA dos estudantes e os OA.

Assim, este trabalho apresenta uma abordagem prática para mapeamento eficaz e automático de características de OA em EA, considerando-se o modelo de Felder e Silverman [11]. O presente trabalho considera o padrão IEEE LOM [18] para metadados de OA, e o relaciona aos EA. A partir desta relação, realiza-se a recuperação e recomendação automática de OA de acordo com os EA de um estudante específico.

Para isto, demonstra-se neste trabalho que a recuperação de OA, dado um modelo de estudante que defina suas preferências de aprendizagem, pode ser automatizada. A principal motivação para este trabalho é a inviabilidade de se recuperar e recomendar manualmente OA 
específicos para cada estudante devido à grande variabilidade de perfis e ao número elevado de OA a serem analisados. Como resultado, obteve-se uma abordagem automática e dinâmica para personalização do ensino em sistemas para ensino à distância com foco no fornecimento automático de OA, considerando-se os EA dos estudantes.

Para tal, realizou-se minuciosa análise sobre as propriedades de cada um dos EA das dimensões do modelo descrito por Felder e Silverman em [11], e um estudo detalhado do padrão IEEE LOM [18], seus campos e propriedades. Com isto, foi possível descobrir quais campos do padrão LOM podem apresentar informações cujos valores se relacionam a estilos de aprendizagem no modelo de Felder e Silverman.

Observou-se nas abordagens analisadas em trabalhos relacionados que estas consideram apenas um subconjunto de EA e geralmente não consideram um padrão de metadados para OA. Desta forma, avançar nestes aspectos é uma grande contribuição deste trabalho, que considera todas as dimensões de EA do modelo de Felder e Silverman, e considera o padrão IEEE LOM como padrão de metadados para OA.

O restante deste trabalho está estruturado da seguinte forma: o capítulo 2 apresenta os conceitos necessários para o entendimento desta abordagem. O capítulo 3 aponta e discute alguns importantes trabalhos relacionados. O capítulo 4 apresenta em detalhes a abordagem proposta. O capítulo 5 demonstra resultados obtidos através dos experimentos realizados. $\mathrm{E}$, finalmente, o capítulo 6 apresenta as considerações finais, e discute as contribuições e trabalhos futuros.

\section{Referencial Teórico}

Nesta sessão, são apresentadas as definições e os conceitos necessários para o entendimento da abordagem proposta, além de citar trabalhos relacionados ao tema desta pesquisa.

\subsection{Estilos de Aprendizagem e Modelo do Es- tudante}

Um modelo de estilos de aprendizagem classifica um estudante de acordo com a forma como este percebe e processa a informação. Um estilo de aprendizagem pode determinar como um indivíduo interage e reage em um ambiente de aprendizagem, refletindo suas preferências reais [40]. Existem diversos modelos que descrevem mo- dos de classificar um estudante em um determinado estilo de aprendizagem [10]. Muitos modelos de estilos de aprendizagem foram propostos, como por exemplo, [9], [11], [17], [19] e [26]. Cada um destes modelos descrevem diferentes aspectos de como estudantes preferem aprender [7].

Em [11] é apresentado um modelo teórico em que cada indivíduo pode se encaixar em diferentes EA em quatro dimensões. Desta forma, cada estudante pode adotar atitudes e comportamentos que se repetem em diferentes momentos e situações [13], [23]. Para satisfazer a diferentes estilos de aprendizagem é necessário que se utilize estratégias de ensino adequadas às várias perspectivas de aprendizagem. Em síntese, EA incorporam as características individuais referentes às tarefas de organizar, perceber, processar, lembrar e pensar para aprender e resolver um problema [40].

Uma característica marcante do modelo de Felder e Silverman [11], e fundamental para este trabalho, é que enquanto a maioria dos modelos classificam estudantes em tipos, este é baseado na ideia de que cada estudante possui uma tendência a um dos estilos em cada uma das suas quatro dimensões. A seguir, apresenta-se resumidamente a descrição das dimensões e estilos de aprendizagem que compõem este modelo:

- Percepção: classifica o estudante de acordo com a forma como o mesmo percebe o conteúdo, podendo ser "sensorial" ou "sensitivo", caso prefira conteúdo abordado de maneira mais concreta, ou "intuitivo", quando há maior preferência por conteúdo mais abstrato;

- Entrada: também definido como "formatoapresentação", esta dimensão indica que um estudante pode ter preferência por informações transmitidas visualmente, sendo, então, considerado "visual", ou informações provindas por texto ou falas, sendo "verbal" ou "textual";

- Processamento: também definido como "participação", indica se o estudante tem atitudes mais ativas em relação ao conteúdo, sendo, portanto, "ativo", ou se o estudante se posiciona mais passivamente, sendo, então, considerado "reflexivo";

- Organização: também definido como "sequência", esta dimensão classifica o estudante de acordo com a forma como conteúdo será exibido, em uma visão progressiva e mais restrita classifica-se em "sequencial", já em uma visão geral e flexível então é "global".

Assim, a Tabela 1 exibe todas as possibilidades de combinação dos EA, totalizando 16 combinações de EA. 
Cada estudante se enquadra melhor em uma ou outra combinação de EA. É fácil notar que recuperar e recomendar manualmente $\mathrm{OA}$ que atendam aos diferentes perfis de estudantes é uma tarefa árdua, devido a diversidade de perfis. Desta forma, recuperar e recomendar automaticamente OA é fundamental para que se obtenha um processo de ensino-aprendizagem efetivamente adaptativo.

As Tabelas 2 e 3 foram criadas a partir da análise das propriedades de cada dimensão descrita em [11] e ilustram esta ideia. A Tabela 2 apresenta uma sumarização da análise realizada para as dimensões Percepção e Entrada da teoria de EA de Felder e Silverman. Já a Tabela 3 resume a análise das dimensões Processamento e Organização. É importante observar o estilo de ensino relacionado a cada EA para que se possa ter uma noção concreta de como deve ser o processo de ensino para diferentes EA.

Ambas as tabelas apresentam pontos importantes concernentes aos EA de cada dimensão, e aos estilos de ensino correspondentes a cada um. Desta forma, é possível se ter uma visão clara sobre as principais diferenças em relação a como o estudante prefere aprender, e como se deve ensinar para atender a cada uma destas preferências.

\begin{tabular}{|l|l|l|l|l|}
\hline Combinações & Percepção & Entrada & Processamento & Organização \\
\hline $\mathbf{1}$ & sensitivo & verbal & ativo & sequencial \\
\hline $\mathbf{2}$ & sensitivo & verbal & ativo & global \\
\hline $\mathbf{3}$ & sensitivo & verbal & reflexivo & sequencial \\
\hline $\mathbf{4}$ & sensitivo & verbal & reflexivo & global \\
\hline $\mathbf{5}$ & sensitivo & visual & ativo & sequencial \\
\hline $\mathbf{6}$ & sensitivo & visual & ativo & global \\
\hline $\mathbf{7}$ & sensitivo & visual & reflexivo & sequencial \\
\hline $\mathbf{8}$ & sensitivo & visual & reflexivo & global \\
\hline $\mathbf{9}$ & intuitivo & verbal & ativo & sequencial \\
\hline $\mathbf{1 0}$ & intuitivo & verbal & ativo & global \\
\hline $\mathbf{1 1}$ & intuitivo & verbal & reflexivo & sequencial \\
\hline $\mathbf{1 2}$ & intuitivo & verbal & reflexivo & global \\
\hline
\end{tabular}

\begin{tabular}{|l|l|l|l|l|}
\hline Combinações & Percepção & Entrada & Processamento & Organização \\
\hline $\mathbf{1 3}$ & intuitivo & visual & ativo & sequencial \\
\hline $\mathbf{1 4}$ & intuitivo & visual & ativo & global \\
\hline $\mathbf{1 5}$ & intuitivo & visual & reflexivo & sequencial \\
\hline $\mathbf{1 6}$ & intuitivo & visual & reflexivo & global \\
\hline
\end{tabular}

Tabela 1 - Todas as 16 possibilidades de combinação de EA [8]

O modelo de Felder e Silverman foi utilizado em diferentes abordagens para sistemas de ensino. Dentre elas destaca-se a abordagem apresentada em [7]. Esta abordagem se diferencia principalmente por apresentar um mecanismo automático e dinâmico para modelagem de EA. Neste processo, os EA de um estudante são descobertos automaticamente, e evoluem ao longo do tempo. Além disto, a abordagem apresentada por [7] considera EA como probabilidades, e não como certezas.

A Tabela 4 apresenta um exemplo de um modelo do estudante, de acordo com a abordagem proposta por [7]. Neste exemplo, os números representam probabilidades do estudante tender a um ou outro estilo dentro de cada dimensão.

Desta forma, no exemplo, o estudante apresenta 35\% de probabilidade em tender ao estilo Ativo, e $65 \%$ de probabilidade em tender ao estilo reflexivo. Uma explicação detalhada de como este modelo é construído pode ser obtida em [7]. Este modelo probabilístico é utilizado como base para recomendação de OA na proposta apresentada neste trabalho.

O modelo é construído de forma automática e dinâmica, evoluindo ao longo do tempo, conforme detalhado em [7] e [8]. Desta forma, é possível iniciar os valores das probabilidades em 0,50 de forma que as probabilidades vão sendo ajustadas evolutivamente por uma abordagem baseada em aprendizagem por reforço. 




Tabela 2 - Análise das dimensões de Felder \& Silverman (Percepção e Entrada) [11]

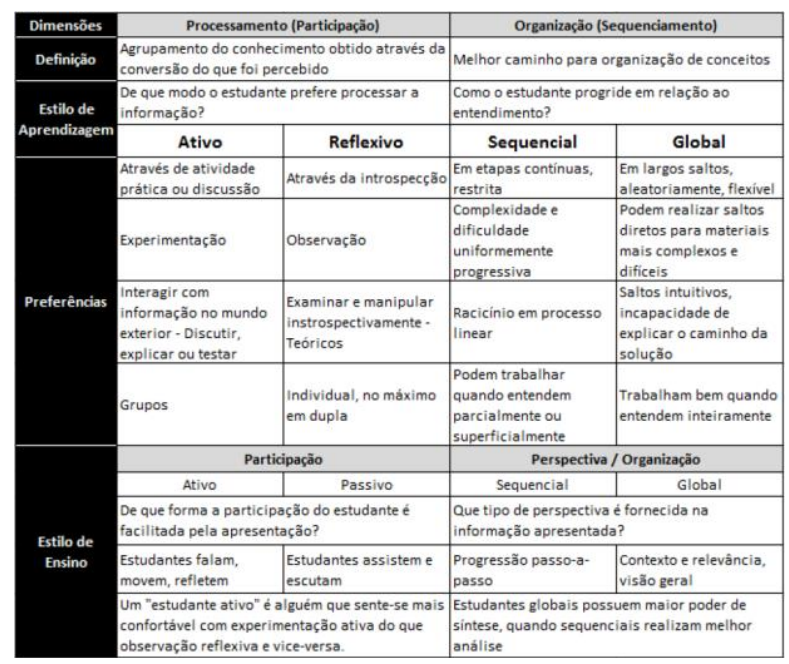

Tabela 3 - Análise das dimensões de Felder \& Silverman (Processamento e Organização) [11]

\begin{tabular}{|c|c|c|c|c|c|c|c|}
\hline \multicolumn{7}{|c|}{ Estilos de Aprendizagem Probabilísticos (EAp) } \\
\hline \multicolumn{2}{|c|}{ Processamento } & \multicolumn{2}{|c|}{ Percepção } & \multicolumn{2}{|c|}{ Entrada } & \multicolumn{2}{c|}{ Organização } \\
\hline Ativo & Reflex. & Sens. & Intuit. & Visual & Verbal & Seq. & Global \\
\hline 0,35 & 0,65 & 0,17 & 0,83 & 0,89 & 0,11 & 0,84 & 0,16 \\
\hline
\end{tabular}

Tabela 4- Modelo do estudante probabilístico em que estilos de aprendizagem são tratados pelo sistema como probabilidades

\subsection{Objetos de Aprendizagem e o padrão IEEE LOM}

Os ambientes de ensino-aprendizagem via web estão sendo cada vez mais utilizados no meio educacional. O desenvolvimento de material didático a ser disponibilizado nesses ambientes exige criatividade, disponibilidade de tempo e conhecimento de tecnologias adequadas. Visando minimizar o tempo e esforço despendido no desenvolvimento de material educacional, e pensando em uma forma de reutilizar esse material, surgiu o conceito de Objetos de Aprendizagem.

Conforme mencionado anteriormente, o padrão IEEE LOM define OA como uma entidade - digital ou não digital - que pode ser usada para ensino, educação ou treinamento. Outros autores complementam esta definição. Segundo [38] são recursos digitais criados para suporte ao ensino, e que permitem a reutilização. Em [21] acrescenta-se ainda que deve-se ter um propósito educacional definido, um elemento que estimule a reflexão do estudante e que ele seja construído de forma que possa ser facilmente reutilizado.

Os conteúdos exibidos ao estudante podem ser organizados de diversas maneiras, uma delas é por meio da catalogação de metadados que descrevam estes conteúdos como OA. Os metadados permitem automatizar a busca e a recuperação de conteúdos e, ao seguirem um padrão bem estabelecido, podem ser reutilizados em diferentes escopos de aprendizagem [40].

Metadados são informações sobre um objeto, seja físico ou digital. Como a oferta de OA é crescente, a falta de informação ou metadados sobre objetos ocupa um ponto crítico e fundamental na habilidade de descobrir, gerenciar e utilizar OA [18].

Os metadados definem o conjunto mínimo de propriedades necessárias para permitir o gerenciamento, a localização e a avaliação destes objetos, utilizados para contextualizar as características de um determinado elemento de informação, no caso os objetos de aprendizagem [5].

Com a popularização dos ambientes virtuais de aprendizagem, materiais didáticos passaram a ser produzidos em larga escala e impulsionaram a organização destes em unidades reusáveis. Com a crescente demanda na utilização desses tipos de recursos, houve a necessidade de estipular uma padronização para a identificação e reutilização desses objetos. Nesse sentido, entidades como IEEE, IMS e ADL (Advanced Distributed Learning), produziram especificações e padrões para suprir 
essas necessidades [32].

O órgão de padronização IEEE [18] definiu um padrão de metadados para OA, o qual é chamado de LOM (Learning Object Metadata). Este é um padrão que descreve OA segundo um modelo universalmente aceito, o que facilita a interoperabilidade entre os diferentes repositórios que empregam o IEEE LOM [39]. Este padrão contém um esquema de dados conceituais que define a estrutura de instâncias de metadados para os OA.

A Tabela 5 ilustra algumas categorias de campos disponíveis no padrão. Dentre as categorias apresentadas, a categoria educacional foi de fundamental importância para este trabalho, justamente por concentrar a descrição das características pedagógicas de um OA.

\begin{tabular}{|l|l|l|}
\hline Categoria LOM & Campos do LOM & Caracterização \\
\hline Geral & $\begin{array}{l}\text { Identificador; tipo; título; } \\
\text { língua; descrição e palavras- } \\
\text { chave; dentre outros. }\end{array}$ & Descrição geral do OA. \\
\hline Técnico & $\begin{array}{l}\text { Formato; tamanho; localiza- } \\
\text { ção; requisitos para uso } \\
\text { (software, hardware, dentre } \\
\text { outros.); dentre outros. }\end{array}$ & $\begin{array}{l}\text { Descrição de características } \\
\text { técnicas e tecnológicas do } \\
\text { OA. }\end{array}$ \\
\hline Educacional & $\begin{array}{l}\text { Tipo de interatividade; tipo de } \\
\text { recurso; grau de dificuldade; } \\
\text { dentre outros. }\end{array}$ & $\begin{array}{l}\text { Descrição das funçães } \\
\text { educacionais e característi- } \\
\text { cas pedagógicas do OA. }\end{array}$ \\
\hline Relacionamento & $\begin{array}{l}\text { Tipo de relacionamento entre } \\
\text { os objetos (linear, rede, } \\
\text { hierárquico, dentre outros) }\end{array}$ & $\begin{array}{l}\text { Descreve o relacionamento } \\
\text { entre OA. }\end{array}$ \\
\hline
\end{tabular}

Tabela 5 - Resumo de alguns campos do IEEE LOM [40]

O IEEE LOM se propõe a facilitar a busca, aquisição, avaliação e utilização de OA por estudantes e professores, ou processos automáticos de software. Permite facilitar o reuso de OA, permitindo o desenvolvimento de repositórios levando em consideração a diversidade cultural, contextos linguísticos nos quais os $\mathrm{OA}$ e seus metadados são reutilizados. Este é um dos esquemas de metadados mais utilizados para descrição de OA [14].

\subsection{Adaptação de conteúdo através da sele- ção personalizada de OA com base em EA}

A Figura 1 apresenta um exemplo de um conceito a ser ensinado em um curso, neste caso o conceito de Servlet, e os OA associados a ele, ou seja, os OA que podem ser utilizados para ensinar este conceito aos diferentes tipos de estudantes. É possível observar que existem dois OA capazes de definir o conceito, dois OA capazes de explicar o conceito, dois OA capazes de exemplificar o conceito, três OA capazes de interpretar o conceito, e dois OA capazes de avaliar o conceito.

Neste caso, o objetivo desta diversidade de OA é que se possa prover adaptação de conteúdo de acordo com os EA do estudante através da seleção de diferentes OA dinamicamente. Desta forma, a recomendação dos OA $(1119 ; 1122 ; 1123 ; 1125 ; 1127 ; 1129 ; 1131 ; 1132 ; 1133)$ poderia atender a um determinado estudante com certas preferências de EA. Já a recomendação dos OA (1120; $1121 ; 1123 ; 1124 ; 1128 ; 1129 ; 1130 ; 1132 ; 1134)$ poderia atender a outro estudante, com uma combinação de EA diferente.

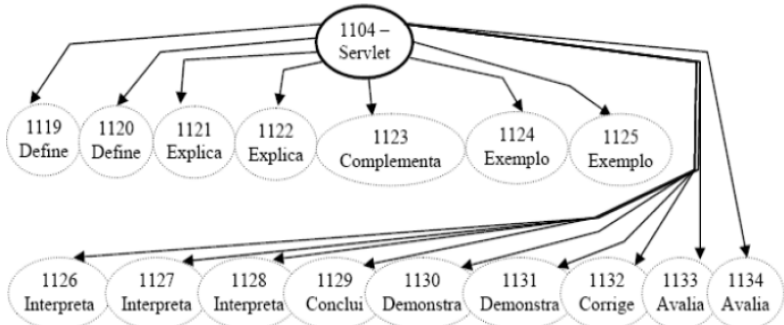

Figura 1 - O conceito de Servlet e respectivos objetos de aprendizagem associados

Então, o ideal é que se tenha uma variabilidade de OA suficiente para ensinar o conceito Servlet de 16 formas diferentes, conforme se apresenta na Tabela 1, levando-se em consideração a composição dos estilos de ensino apontados nas Tabelas 2 e 3 . Como pode-se perceber, em um curso com muitos conceitos a serem ensinados, muitos $\mathrm{OA}$ são necessários para atender aos vários perfis de estudantes, ficando então bastante clara a importância de mecanismos automáticos e dinâmicos para recuperação e recomendação de $\mathrm{OA}$ com base em EA.

Desta forma, faz-se necessária a associação automática e dinâmica de OA aos conceitos a serem ensinados, permitindo que se leve em consideração o nível cognitivo e os EA dos estudantes, obtendo-se um processo automático e dinâmico para fornecimento de adaptabilidade em sistemas para $\mathrm{EaD}$.

A Figura 2 apresenta parte de um mapa conceitual simplificado que modela o domínio de um curso de Programação Orientada a Objetos, em que se tem a representação dos relacionamentos entre alguns dos principais conceitos do curso.

A ideia é que se possa, de forma automática, adaptar o ensino de cada um destes conceitos para cada um dos 
16 perfis apresentados na Tabela 1 . Para isto, é necessário que se possa associar a cada um destes conceitos uma diversidade de OA, como apresentado na Figura 1, de forma que a adaptação do ensino seja efetiva. Neste contexto, observa-se que a recuperação automática de $\mathrm{OA}$ em repositórios é fundamental para que seja possível a adaptação do ensino aos diversos EA.

De acordo com [8], padrões de metadados para indexação de OA, tais como o LOM, são de extrema importância na reutilização desse conteúdo e, também, facilitam a definição de regras de navegação condicionais e adaptativas levando-se em consideração as características presentes no modelo do estudante.

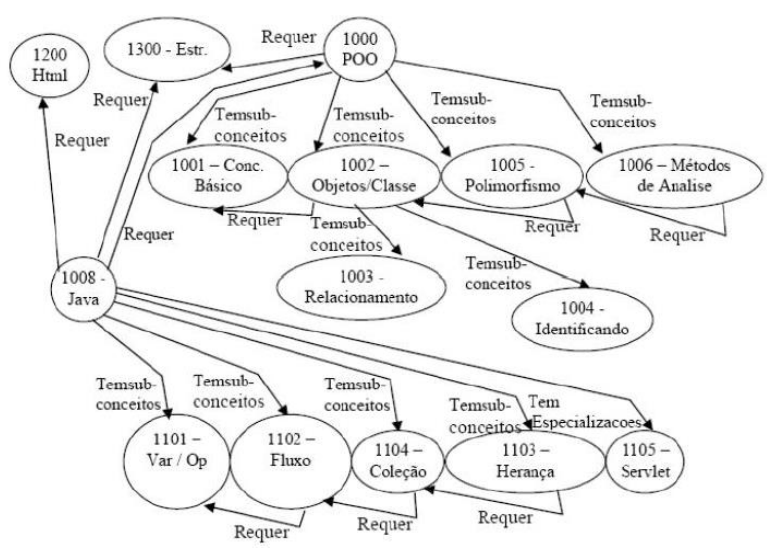

Figura 2 - Parte do mapa conceitual de um curso de Programação Orientada a Objetos

\section{Trabalhos Relacionados}

Alguns trabalhos com abordagens relacionadas podem ser encontrados em [20] [22] [28] [29] [30] [31] [40]. Dentre esses trabalhos, [28] apresenta um componente que sugere links para personalizar a interação do usuário baseado em algoritmos de mineração de dados que avaliam o $\log$ do estudante. O componente foi integrado ao projeto AHA! que é um ambiente virtual de aprendizagem que adota os conceitos de hipermídia adaptativa para o oferecimento de conteúdo aos alunos. As recomendações são realizadas com base em links cadastrados para o curso. Porém, a relação entre os OA e os perfis não é realizada de maneira automatizada, sendo necessário que o tutor realize a correlação manualmente no momento do cadastro dos objetos [40].
O trabalho apresentado em [22] constrói cenários de aprendizagem de acordo com o modelo de Kolb [19], que relaciona o EA com conteúdos de aprendizagem por meio do padrão SCORM (Shareable Content Object Reference Model) [12]. Embora trabalhe com padrões para especificação de conteúdos, a proposta não faz a associação do estilo de aprendizagem usando categorização de elementos [40].

O SEDHI (Sistema Educacional Hipermídia) [30] foi projetado para ser usado em cursos à distância na web. Para tanto, ele realiza a recomendação de conteúdos com base no perfil do estudante na dimensão de seu conhecimento (iniciante, intermediário, ou avançado). O sistema realiza um acompanhamento das atividades realizadas pelo estudante e, com base nestas e em seu perfil, classifica o estudante. É a partir desta classificação que o sistema realiza a adaptação da navegação no curso empregando as técnicas de ocultamento e anotação de links. Não há correlação automática entre os links e os estilos de aprendizagem [40].

É importante observar que alguns estudos realizam análises sobre quais tipos de objetos de aprendizagem e mídias eletrônicas são mais propícios a um determinado EA. Em [31] observou-se que algumas ações instrucionais são mais apropriadas a um EA do que outras, e atribuiu-se valores no intervalo $[0,1]$ a cada ação instrucional, indicando o quanto tal ação satisfaz um EA. Em [20] apresenta-se um exemplo de aplicação desta política de associação entre ações instrucionais e EA. Ainda, em [29] classifica-se os diferentes tipos de OA de acordo com o valor do atributo Learning Resource Type do IMS Metadata Standard. Logicamente tal associação não é trivial, e deve contar com especialistas em educação, pedagogia e psicologia cognitiva.

Em [1] apresenta-se alguns modelos e ferramentas para a construção de sistemas educacionais adaptativos e semânticos, descrevendo-se uma arquitetura multicamadas e um conjunto de ontologias e regras de mapeamento para ser usada na construção deste tipo de sistema. Também, [3] apresenta a implementação de um módulo de visualização de conteúdos adaptados de acordo com o estilo de aprendizagem de cada estudante, gerando materiais diferenciados de acordo com seus perfis.

A abordagem de pesquisa que mais se assemelha a este trabalho é apresentada em [40]. Por isto, esta é descrita com maiores detalhes. Nela, é definida a metodologia e-LORS, em que os EA são organizados de acordo com dimensões que incorporam as preferências de aprendizagem de um estudante. Seu objetivo é verificar o relacionamento entre os EA e os OA, processando-se os 
metadados que os descrevem. Torna-se possível, então, a recomendação dos $\mathrm{OA}$ que mais bem correspondem aos EA de um determinado estudante. Para a tarefa de descrição dos perfis de aprendizagem em dimensões discretas foi adotado o modelo de estilos de aprendizagem de Felder e Silverman.

As diferenças determinantes entre este trabalho e [40] consiste no fato de que o segundo considera apenas os campos "Tipo de Interatividade" e "Tipo de Recurso de Ensino" do LOM para realizar as recomendações. Além disto, os OA recomendados têm pesos equivalentes em relação ao quanto estão em consonância com o perfil do estudante, o que ocorre diferentemente neste trabalho, já que mais campos são utilizados, e os OA fornecidos possuem diferentes níveis de relevância em relação às preferências do estudante (conforme descrito na próxima seção).

Finalmente, a recuperação automática de OA na web, através de seus metadados, levando-se em consideração características específicas de EA é um importante campo de pesquisa. De acordo com [8], a reutilização em larga escala de repositórios de $\mathrm{OA}$ e a interoperabilidade em ambientes virtuais de aprendizagem tem sido possibilitadas por tecnologias da Web Semântica [4], que tem sua base na eXtensible Markup Language (XML) [35] e em padrões para representação de conhecimento tais como o Resource Description Framework (RDF) [34] e a Web Ontology Language (OWL) [33]. A busca e manipulação deste conhecimento intercambiável é facilitada por linguagens de consulta, tal como a XQuery [36].

\section{Abordagem Proposta}

Esta seção apresenta em detalhes a abordagem proposta neste trabalho. A abordagem apresentada neste trabalho define relações entre características de EA e os campos do LOM. Para isto, o desenvolvimento desta abordagem seguiu a metodologia de trabalho apresentada a seguir:

1. Estudo e análise das propriedades dos estilos de aprendizagem considerando-se a teoria de Felder e Silverman.

2. Estudo e análise dos campos e valores dos metadados no padrão IEEE LOM.

3. Determinação de quais campos do LOM possibilitam identificar o estilo de ensino característico do OA.

4. Mapeamento da inter-relação entre estes campos, e seus valores, e os EA no modelo de Felder e Silverman.
5. Implementação computacional das associações descobertas através de regras de produção.

6. Implementação de um algoritmo para recomendação automática de OA de acordo com os EA do estudante, levando-se em consideração a ordem de relevância dos OA para o perfil do estudante.

7. Implementação de um protótipo para realização de experimentos.

Considerando-se as características de EA sumarizadas nas Tabelas 2 e 3, e detalhadas em [11], foram verificados os campos do padrão IEEE LOM [18] cujos conteúdos tinham ligação com as características dos EA do modelo de Felder e Silverman. Assim, foi definido quais destes campos seriam utilizados para realizar o mapeamento entre o modelo do estudante e metadados de OA.

As Tabelas 6 a 10 apresentam os campos do LOM que tiveram seus valores mapeados aos EA no modelo de Felder e Silverman neste trabalho. Para cada dimensão do modelo apresenta-se o EA que é satisfeito para determinado campo e valor do LOM. Assim, pode-se ter uma ideia clara da relação entre metadados LOM e os EA.

\begin{tabular}{|l|l|c|c|c|c|}
\hline Campo & Valor & Percepção & Entrada & Processamento & Organização \\
\hline $\begin{array}{l}\mathbf{1 . 7} \text { - Estrutu- } \\
\text { ra }\end{array}$ & rede & - & - & - & $\begin{array}{c}\text { Global (visão } \\
\text { geral) }\end{array}$ \\
\cline { 2 - 6 } & linear & - & - & - & $\begin{array}{c}\text { Sequencial } \\
\text { (etapas } \\
\text { contínuas) }\end{array}$ \\
\hline
\end{tabular}

Tabela 6 - Campo Estrutura (Structure) do LOM com seus valores mapeados aos EA no modelo de Felder e Silverman

\begin{tabular}{|l|l|c|c|c|c|}
\hline Campo & Valor & Percepção & Entrada & Processamento & Organização \\
\hline 4.1 - Formato & áudio & $\begin{array}{c}\text { Sensitivo (coleta } \\
\text { de dados por meio } \\
\text { dos sentidos: } \\
\text { audição) }\end{array}$ & $\begin{array}{c}\text { Verbal (pala- } \\
\text { vras, sons) }\end{array}$ & $\begin{array}{c}\text { Reflexivo } \\
\text { (documentos } \\
\text { expositivos, } \\
\text { aprendizagem } \\
\text { passiva) }\end{array}$ & - \\
\cline { 2 - 6 } & imagem & $\begin{array}{c}\text { Sensitivo } \\
\text { (percepcão através } \\
\text { dos sentidos: } \\
\text { visão) }\end{array}$ & $\begin{array}{c}\text { Visual (sinais, } \\
\text { figuras) }\end{array}$ & $\begin{array}{c}\text { Reflexivo } \\
\text { (aprendizagem } \\
\text { passiva) }\end{array}$ & - \\
\cline { 2 - 6 } & texto & $\begin{array}{c}\text { Intuitivos (são } \\
\text { mais confortáveis } \\
\text { com símbolos) }\end{array}$ & $\begin{array}{c}\text { Verbal (pala- } \\
\text { vras) }\end{array}$ & $\begin{array}{c}\text { Reflexivo } \\
\text { (aprendizagem } \\
\text { passiva) }\end{array}$ & - \\
\cline { 2 - 6 } & $\begin{array}{c}\text { Sensitivo } \\
\text { (percepcão através } \\
\text { dos sentidos: } \\
\text { visão e audição) }\end{array}$ & $\begin{array}{c}\text { Visual, verbal } \\
\text { (imagem e som) }\end{array}$ & $\begin{array}{c}\text { Reflexivo } \\
\text { (documentos } \\
\text { expositivos) }\end{array}$ & - \\
\hline
\end{tabular}

Tabela 7 - Campo Formato (Format) do LOM com seus valores mapeados aos EA no modelo de Felder e Silverman

\begin{tabular}{|l|l|c|c|c|c|}
\hline Campo & Valor & Percepção & Entrada & Processamento & Organização \\
\hline $\begin{array}{l}\mathbf{5 . 1} \text { - Tipo de } \\
\text { Interatividade }\end{array}$ & ativo & $\begin{array}{c}\text { Sensitivo (são } \\
\text { práticos, com } \\
\text { preferência por } \\
\text { material concreto }\end{array}$ & - & $\begin{array}{c}\text { Ativo (conteúdo } \\
\text { que induz direta- } \\
\text { mente a ação } \\
\text { produtiva, aprender }\end{array}$ & - \\
\hline
\end{tabular}




\begin{tabular}{|l|l|c|c|c|c|}
\hline & $\begin{array}{c}\text { e aplicações no } \\
\text { mundo real) }\end{array}$ & fazendo) & \\
\cline { 2 - 6 } & expositivo & $\begin{array}{c}\text { Intuitivo (prefe- } \\
\text { rência por } \\
\text { material terórico e } \\
\text { abstrato) }\end{array}$ & - & $\begin{array}{c}\text { Reflexivo } \\
\text { (trabalho do aluno } \\
\text { consiste em } \\
\text { absorver o } \\
\text { conteúdo exposto) }\end{array}$ & - \\
\cline { 2 - 6 } & misto & $\begin{array}{c}\text { Sensitivo e } \\
\text { intuitivo }\end{array}$ & - & Ativo e reflexivo & - \\
\hline
\end{tabular}

Tabela 8 - Campo Tipo de Interatividade (Interactivity type) do LOM com seus valores mapeados aos EA no modelo de Felder e Silverman

\begin{tabular}{|c|c|c|c|c|c|}
\hline Campo & Valor & Percepção & Entrada & Processamento & Organização \\
\hline \multirow[t]{10}{*}{$\begin{array}{l}5.2 \text { - Tipo de } \\
\text { recurso de } \\
\text { aprendizagem }\end{array}$} & exercício & - & - & $\begin{array}{l}\text { Ativo (estudante } \\
\text { deve encontrar } \\
\text { uma solução, } \\
\text { aprender fazendo) }\end{array}$ & - \\
\hline & simulação & $\begin{array}{c}\text { Sensitivo (são } \\
\text { práticos, com } \\
\text { preferência por } \\
\text { fatos concretos e } \\
\text { aplicações no } \\
\text { mundo real) }\end{array}$ & - & $\begin{array}{l}\text { Ativo (manipula- } \\
\text { ção, controle, } \\
\text { entrada de dados, } \\
\text { aprender experi- } \\
\text { mentando) }\end{array}$ & - \\
\hline & questionário & - & $\begin{array}{l}\text { Verbal (pergun- } \\
\text { tas, palavras) }\end{array}$ & $\begin{array}{l}\text { Ativo (estudante } \\
\text { deve escolher } \\
\text { dentre várias } \\
\text { opções) }\end{array}$ & - \\
\hline & diagrama & $\begin{array}{c}\text { Sensitivo } \\
\text { (informaçãoo } \\
\text { concreta, prática e } \\
\text { processual) }\end{array}$ & $\begin{array}{l}\text { Visual (sinais, } \\
\text { figuras, } \\
\text { símbolos) }\end{array}$ & $\begin{array}{c}\text { Reflexivo } \\
\text { (aprendizagem } \\
\text { passiva) }\end{array}$ & - \\
\hline & figura & $\begin{array}{c}\text { Sensitivo } \\
\text { (informação } \\
\text { concreta, prática e } \\
\text { processual) }\end{array}$ & $\begin{array}{l}\text { Visual (sinais, } \\
\text { figuras, } \\
\text { símbolos) }\end{array}$ & $\begin{array}{l}\text { Reflexivo } \\
\text { (aprendizagem } \\
\text { passiva) }\end{array}$ & - \\
\hline & gráfico & $\begin{array}{c}\text { Sensitivo } \\
\text { (informação } \\
\text { concreta, prática e } \\
\text { processual) }\end{array}$ & $\begin{array}{l}\text { Visual (sinais, } \\
\text { figuras, } \\
\text { símbolos) }\end{array}$ & $\begin{array}{c}\text { Reflexivo } \\
\text { (aprendizagem } \\
\text { passiva) }\end{array}$ & - \\
\hline & narrativa & - & $\begin{array}{l}\text { Verbal (expla- } \\
\text { nação verbal) }\end{array}$ & $\begin{array}{l}\text { Reflexivo } \\
\text { (aprendizagem } \\
\text { passiva) }\end{array}$ & - \\
\hline & problema & $\begin{array}{c}\text { Intuitivo (inova- } \\
\text { ção, novos } \\
\text { conceitos, } \\
\text { complexidade, } \\
\text { desafio, criativi- } \\
\text { dade) }\end{array}$ & $\begin{array}{l}\text { Verbal (expla- } \\
\text { nação verbal) }\end{array}$ & $\begin{array}{l}\text { Ativo (estudante } \\
\text { deve encontrar a } \\
\text { solução) }\end{array}$ & - \\
\hline & $\begin{array}{l}\text { auto- } \\
\text { avaliação }\end{array}$ & $\begin{array}{l}\text { Intuitivo (interno, } \\
\text { insight) }\end{array}$ & $\begin{array}{l}\text { Verbal (expla- } \\
\text { nação verbal) }\end{array}$ & $\begin{array}{l}\text { Ativo (manipula- } \\
\text { ção, controle, } \\
\text { entrada de dados) }\end{array}$ & - \\
\hline & palestra & - & $\begin{array}{l}\text { Verbal (expla- } \\
\text { nação verbal) }\end{array}$ & $\begin{array}{l}\text { Reflexivo } \\
\text { (aprendizagem } \\
\text { passiva) }\end{array}$ & - \\
\hline
\end{tabular}

Tabela 9 - Campo Tipo de Recurso (Resource type) de Aprendizagem do LOM com seus valores mapeados aos EA no modelo de Felder e Silverman

\begin{tabular}{|c|c|c|c|c|c|}
\hline Campo & Valor & Percepção & Entrada & Processamento & Organização \\
\hline \multirow[t]{2}{*}{$\begin{array}{l}5.8 \text { - Dificul- } \\
\text { dade }\end{array}$} & Simples/ fácil & $\begin{array}{c}\text { Sensitivo } \\
\text { (padronização, } \\
\text { simplicidade, } \\
\text { memorização de } \\
\text { fatos, concreto) }\end{array}$ & - & - & - \\
\hline & $\begin{array}{l}\text { difíicil } \\
\text { /complexo }\end{array}$ & $\begin{array}{l}\text { Intuitivo (inova- } \\
\text { ção, novos } \\
\text { conceitos, } \\
\text { complexidade, } \\
\text { capacidade de } \\
\text { abstração) }\end{array}$ & - & - & - \\
\hline
\end{tabular}

Tabela 10 - Campo Dificuldade (Dificulty) do LOM com seus valores mapeados aos EA no modelo de Felder e Silverman

Nas Tabelas 6 a 10, o EA atendido por determinado valor de um campo do LOM é descrito na coluna da di- mensão correspondente, seguido por justificativa entre parênteses. A fim de explicar alguns relacionamentos, seguem alguns detalhes.

O campo "estrutura" (structure) preenchido com valor "rede" é mapeado para o estilo global da dimensão organização. Esta característica do OA fornece ao estudante uma síntese do conteúdo nele contido, o que é uma particularidade marcante do estudante com tendência a este EA.

O padrão IEEE LOM define como documentos ativos, aqueles onde o conteúdo induz diretamente ação produtiva por parte do aluno. Um OA com "tipo de interatividade" ativo incita a ação produtiva ou decisão por parte do aluno [18], características que são correspondentes ao perfil de um estudante com o estilo ativo da dimensão processamento, já que estes se sentem, segundo [11], mais confortáveis com a experimentação ativa. São exemplos de documentos ativos: simulação, questionário, exercício e enunciado de problema [18].

Equivalente a estas definições, há documentos definidos como expositivos, em que o trabalho do aluno consiste principalmente em absorver o conteúdo exposto a ele, geralmente através de texto, imagens ou som. Um OA expositivo apresenta informações, mas não incita o aluno por nenhuma entrada semanticamente significativa [18]. Estas são características pertinentes ao estilo reflexivo da dimensão processamento, pois tem como traços marcantes a observação reflexiva e passividade na interação com o OA. São exemplos de documentos expositivos: ensaios, clipes de vídeo, todos os tipos de material gráfico, e documentos de hipertexto.

O modelo proposto neste artigo foi especificado de maneira que possa ser utilizado por diferentes sistemas de ensino. $\mathrm{O}$ mapeamento de objetos é realizado com base no tema de estudo a ser apresentado e os EA do estudante. Foi implementado um protótipo para testes e avaliação empírica do modelo, o que possibilitou a verificação e validação do funcionamento do mapeamento de características de OA em características de EA para fornecimento automático de adaptatividade em sistemas para $\mathrm{EaD}$.

No protótipo, o modelo do estudante composto por distribuições de probabilidades, conforme explica [7], [8], é fornecido pelo usuário, de forma que os OA sejam recomendados de acordo com os EA de um estudante fictício. Na prática, conforme mencionado anteriormente, o modelo do estudante deve ser construído de maneira automática e dinâmica, conforme apresentado em [8]. É possível também informar o assunto desejado para que se 
possa recomendar apenas os $\mathrm{OA}$ que tratem de um tema de interesse específico.

Então, inicialmente, realiza-se uma filtragem, buscando-se no repositório quais $\mathrm{OA}$ possuem a ocorrência dos termos que foram inseridos no campo assunto da interface do protótipo desenvolvido. Compara-se tais termos com aqueles presentes nos campos Título, Descrição e Palavras-chaves. O resultado deste filtro é um subconjunto dos OA que tratam apenas do conteúdo desejado (esta etapa do processo foi baseada na primeira fase do sistema de recomendação e-LORS [40]).

A partir disto, o sistema recomenda automaticamente OA cujos metadados apresentam algum nível de relevância ao perfil inserido pelo usuário. $\mathrm{O}$ resultado final da execução é uma lista de OA ordenados pela relevância em relação aos EA do estudante. Os mais relevantes aparecem primeiro na lista.

O cálculo da relevância é bem simples. Soma-se o valor das relevâncias obtidas pelo OA em cada uma das quatro dimensões de EA. A relevância em uma dimensão é calculada da seguinte forma: considerando-se as Tabelas 6 a 10, para cada EA satisfeito pelos valores dos metadados do OA, soma-se o valor referente ao EA presente no modelo do estudante (vide Tabela 4).

Por exemplo, considerando-se o modelo do estudante apresentado na Tabela 4, se os valores dos metadados de um determinado OA atendem ao EA Reflexivo em 1 campo, o EA Sensitivo em 2 campos, ao EA Visual em 2 campos e ao EA Global em 1 campo, temos a seguinte relevância para este $\mathrm{OA}$ em relação aos EA do estudante apresentado:

$$
(1 \times 65)+(2 \times 17)+(2 \times 89)+(1 \times 16)=293
$$

Então, basicamente, realiza-se a soma dos valores dos EA do estudante a cada vez que o EA é atendido pelos valores dos campos do OA. Então, de acordo com as Tabelas 6 a 10, a relevância de um OA para o estilo sensitivo é definida verificando-se se o campo "Formato" possui o valor "áudio", "imagem", ou "vídeo"; se o campo "Tipo de interatividade" possui o valor "ativo" ou "misto"; se o campo "tipo de recurso de aprendizagem" possui o valor "simulação", "diagrama", "figura" ou "gráfico"; se o campo "Dificuldade" possui o valor "simples/fácil". A cada assertiva verdadeira, o valor da probabilidade do EA é somado à relevância do OA.

Desta forma, o cálculo da relevância R para um OA é dado por (1). Em (1), $\mathrm{Q}_{\mathrm{i}}$ representa a quantidade de campos dos metadados do OA que o EA satisfaz, e EA representa o valor da probabilidade de preferência ao EA pelo estudante, armazenado no modelo do estudante (como exemplificado na Tabela 4). É importante observar que são contabilizados os oito EA presentes no modelo do estudante (ativo, reflexivo, sensitivo, intuitivo,

$$
R=\sum_{i=1}^{8}\left(Q_{i} \times E A_{i}\right)(1)
$$

visual, verbal, sequencial e global). Por isto o índice $i$ varia de 1 a 8 .

Como o cálculo da relevância dos $\mathrm{OA}$, e consequentemente a recomendação dos mesmos, depende dos valores probabilísticos armazenados no modelo do estudante, a medida que estes valores sofrem alterações, o resultado do processo de recomendação também é alterado. O processo de atualização destes valores é automático e dinâmico, e está detalhado em [7], [8]. A próxima seção apresenta alguns experimentos e os resultados obtidos.

\section{Experimentos e Resultados}

Exemplos de OA com metadados catalogados no padrão IEEE LOM podem ser obtidos no LOM Demonstration collection, disponível em $<\mathrm{http}: / /$ http://www.nzdl.org/gsd/>, e foram utilizados nos experimentos apresentados nesta seção.

Tais exemplos de OA foram inseridos manualmente no repositório, mantendo o idioma inglês original em que estas amostras estão disponíveis. Contudo, estes exemplos de OA não possuem todos os campos preenchidos. Portanto, alguns valores fictícios foram acrescentados àqueles que se encontravam em branco. Com isto, foi possível obter-se os metadados necessários para a realização de experimentos com a abordagem proposta.

Para execução dos experimentos, o repositório de OA foi simulado através de um banco de dados relacional. Foram executados experimentos utilizando-se modelos de EA probabilísticos, conforme propõem [7], [8]. Para todos os experimentos, o assunto inserido para filtragem foi "Ciências e Artes" (Sciences and Arts, em inglês), pois os exemplos de OA encontrados no LOM Demonstration collection estão relacionados a este tema, e no idioma inglês. A interface do protótipo para realização dos experimentos é apresentada na Figura 3. 




Figura 3 - Tela inicial do protótipo implementado

É possível observar na Figura 3 os campos nome, assunto e os valores dos EA do estudante, conforme a proposta apresentada em [8]. Neste caso, as preferências do estudante são representadas por probabilidades de se preferir um ou outro estilo dentro de cada dimensão, conforme mencionado anteriormente.

Após realizar-se a entrada dos EA do estudante, aciona-se o botão "Mapear", que após executar o cálculo da relevância dos OA encontrados, exibe no painel "Estilo de Aprendizagem" a distribuição probabilística do modelo do estudante. Logo em seguida, o painel "Objetos de Aprendizagem" contendo os OA ordenados pela relevância é apresentado, conforme Figura 4.

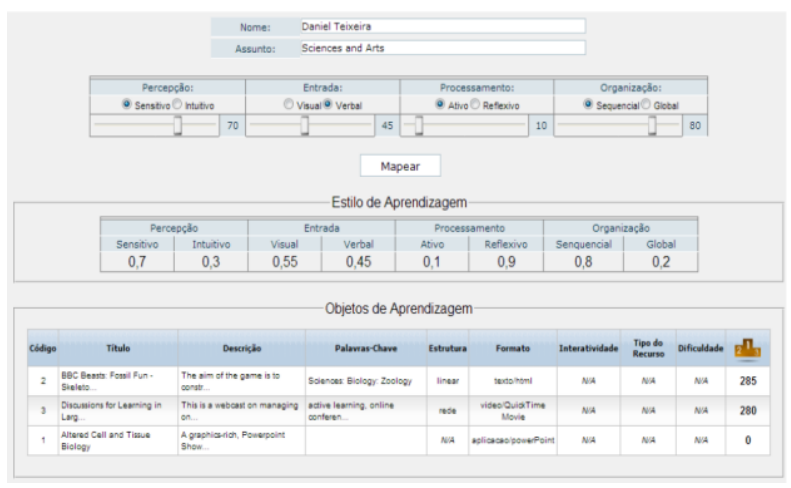

Figura 4 - Exemplo de resultado de recomendação

\subsection{Primeiro Experimento}

Utilizando os valores probabilísticos de modelagem do estudante conforme exibido na Figura 5, têm-se, na Figura 6, os OA recomendados para este perfil, devidamente ordenados pela relevância do $\mathrm{OA}$ em relação aos EA do estudante.

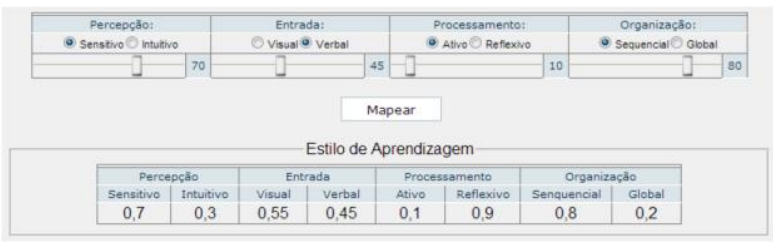

Figura 5 - EA inserido para primeiro experimento

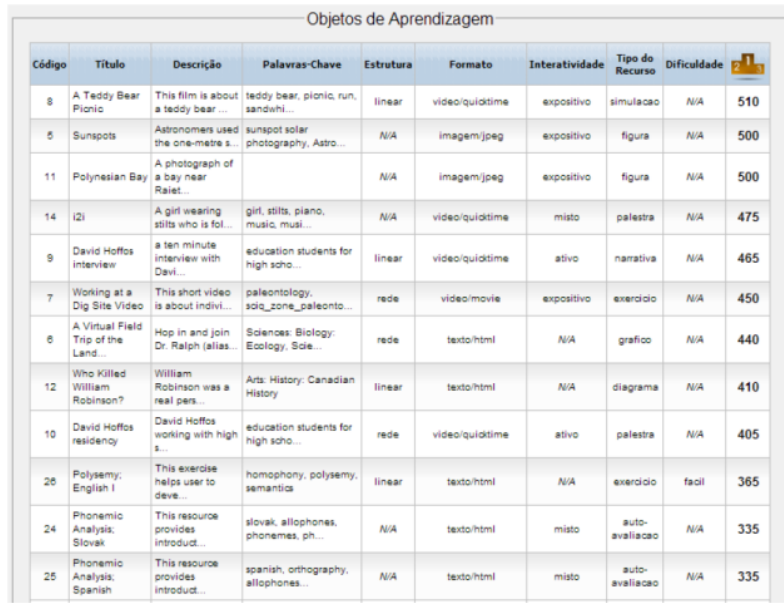

Figura 6 - Recomendação de OA resultante do primeiro experimento

Com relação ao resultado obtido, tem-se o OA registrado no repositório com código "8" como o objeto mais aderente ao perfil do estudante inserido. Isto porque o mesmo apresentou uma relevância total de " 510 ", que consiste na soma das tendências que o mesmo obteve para cada dimensão, de acordo com as Tabelas 6 a 10.

Observe que para o EA "visual" o campo "Formato" do tipo "vídeo" atende a dimensão e soma " 55 " à relevância do OA, devido à probabilidade visual inserida no modelo do estudante. Para o estilo "verbal", o valor "vídeo" no campo "Formato" também apresenta correlação, e assim a relevância é acrescida em "45", que se refere à probabilidade de preferência do estudante por este EA.

Para o estilo "ativo", a relevância é acrescida em "10", devido ao campo "Tipo de Recurso" possuir o valor "simulação". A relevância é analisada e contabilizada para cada EA. O mesmo processo é realizado para todos os OA obtidos.

\subsection{Segundo Experimento}

O modelo de estudante utilizado neste experimento é apresentado na Figura 7. Em relação ao primeiro experimento, o estudante passa a possuir característica "global" com probabilidade maior do que "sequencial".

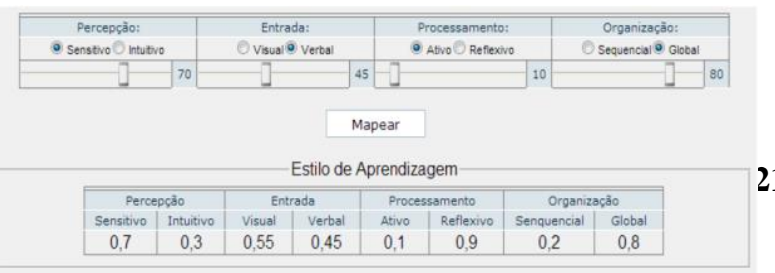


Figura 7 - EA inserido para segundo experimento

Esta mudança de perfil altera a ordenação dos OA em relação ao experimento 1 , conforme observa-se na Figura 8. Neste experimento, o OA que mais atende aos EA do estudante é aquele de código "7". O que leva a este fato é o seu metadado "Estrutura" com valor "rede", que está em consonância com a preferência pelo EA "global", acrescentando o valor " 80 " em sua relevância.

O OA com código " 8 ", que fora o mais indicado no experimento anterior, passa a ser o quinto mais relevante. O OA perdeu significativamente sua relevância, pois possui característica linear, sendo neste caso o estudante fortemente global.

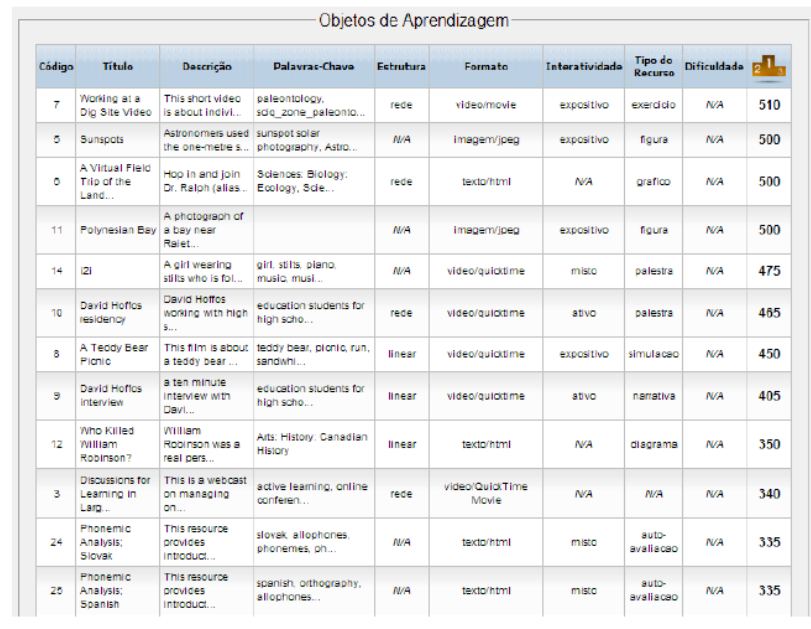

Figura 8 - Recomendação de OA resultante do segundo experimento

\subsection{Terceiro Experimento}

Para este experimento, em relação ao anterior, o perfil do estudante inverte os valores para a dimensão Processamento, e passa a ter a distribuição de " 90 " para o extremo ativo e "10" para o reflexivo, conforme Figura 9.

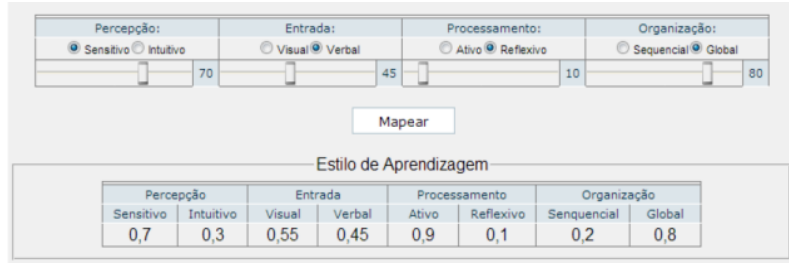

Figura 9 - EA inserido para terceiro experimento
Tal alteração faz com que o OA de código " 10 ” passe a ser o objeto mais recomendado ao estudante, conforme apresenta a Figura 10. Essa mudança na recomendação em relação ao experimento anterior deve-se ao fato de que o OA de código "7" (melhor recomendado no experimento anterior) perdeu relevância por apresentar característica reflexiva, sendo neste caso o estudante fortemente ativo. Desta forma, a probabilidade de preferência de "90" para o EA ativo no experimento atual faz com que a característica ativa no campo "tipo de interatividade" do OA "10" some maior relevância.

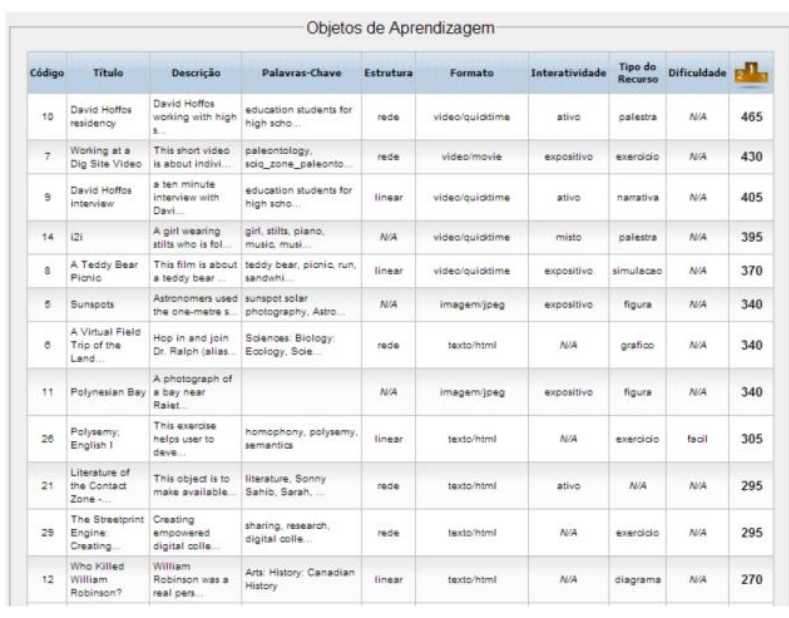

Figura 10 - Recomendação de OA resultante do terceiro experimento

A análise dos resultados obtidos nesta seção nos permite concluir que a abordagem proposta satisfaz aos objetivos inicialmente propostos, podendo ser implantada e utilizada em LMS existentes. A próxima seção apresenta algumas conclusões e trabalhos futuros.

\section{Conclusão e trabalhos futuros}

Os experimentos da abordagem proposta apresentaram resultados promissores, conforme evidenciado no capítulo anterior. Os experimentos realizados apresentaram a recomendação de OA de maneira eficaz, levando em consideração a correlação com a combinação de EA com valores probabilísticos para os polos de cada dimensão. Como os EA não permanecem constantes ao longo do tempo, percebe-se que este processo tem caráter não determinístico [8], pois são atualizados de forma automática e dinâmica ao longo do processo de ensinoaprendizagem.

A validação da abordagem proposta por meio de um 
protótipo para experimentação em que pode-se fornecer livremente os EA do estudante foi fundamental para a conclusão deste trabalho, haja vista o quanto de recurso e tempo seria necessário para a implantação e teste da abordagem em um ambiente e-learning.

Portanto, é necessário que se avalie modelos antes de utilizá-lo em um LMS, devido à complexidade que envolve este tipo de projeto. Esta necessidade torna-se ainda mais evidente ao se analisar a demanda por este tipo de recurso [16].

As principais contribuições deste trabalho foram a obtenção de uma abordagem eficaz para mapear características de OA em características de EA de maneira dinâmica e automática, de modo a fornecer adaptatividade. Isto comprova a possibilidade de se automatizar o relacionamento entre metadados de OA e propriedades de EA, quando estes estão utilizando o padrão IEEE LOM e o modelo de Felder e Silverman [11], respectivamente.

Além disto, um resultado importante foi a ordenação dos OA de acordo com a relevância em relação ao perfil do estudante, sendo que a modelagem probabilística de EA utilizada foi de fundamental importância para esta abordagem.

O principal desafio encontrado foi o estabelecimento da relação entre EA e LOM, apresentado pelas Tabelas 6 a 10. Este mapeamento demandou grande esforço em pesquisa, demandando estudo e análise exaustiva de amplo conteúdo.

Como possíveis trabalhos futuros, têm-se inicialmente a utilização de mais campos do padrão IEEE LOM para filtragem, como por exemplo, os campos "Idioma", "Cobertura" (demográfica), "Intervalo de Faixa Etária", que necessitam de um perfil mais detalhado, mas que poderiam fornecer aos estudantes OA com características ainda mais aderentes ao perfil de cada um.

Em um segundo momento, este trabalho será aplicado em um Sistema de Gerenciamento de Aprendizagem, atuando com a abordagem apresentada em [8], recebendo a modelagem probabilística do estudante e retornando os OA que mais se adaptam a este perfil. Então, será realizada a avaliação da adequação do modelo proposto aos interesses dos estudantes, pela possibilidade de haver inconsistências entre os OA recomendados, após a execução do módulo de software, e o que estes estão buscando em dado momento de seu percurso de aprendizagem.

\section{Referências}

[1] BITTENCOURT, I.; COSTA, E. "Modelos e Ferramentas para a Construção de Sistemas Educacionais adaptativos e Semânticos". Revista Brasileira de Informática na Educação, Volume 19, Número 1, 2011.

[2] BROOKS, C.; Greer, J.; MELIS, E. \& ULLRICH, C. "Combining its and e-learning technologies opportunities and challenges". Proceedings of the 8th international conference on Intelligent Tutoring Systems, Springer-Verlag, 2006, 278-287.

[3] CANDOTTI, C. T., GELLER, M., SILVEIRA, S. R., MARQUES , E. M., SANTANA, M. B. "Sistema de hipermídia adaptativo baseado em estilos cognitivos". RENOTE - Revista Novas Tecnologias na Educação ISSN 1679-1916, 4(2), 2006.

[4] DAVIES, J.; "Semantic Web technologies : trends and research in ontology-based systems". John Wiley \& Sons Ltd, 2006.

[5] DEVEDŽIC, V.; GAŠEVIC, D.; DJURIC, D.; "Clarifying the meta". In International Journal of Information and Communication Technology, 2008.

[6] DORÇA, F. A.; LIMA, L. V.; FERNANDES, M. A. ; LOPES, C. R. "A New Approach to Discover Students Learning Styles in Adaptive Educational Systems". Revista Brasileira de Informática na Educação, Volume 21, Número 1, 2013.

[7] DORÇA, F. A. ; LIMA, L. V. ; FERNANDES, M. A. ; LOPES, C. R. "Automatic student modeling in adaptive educational systems through probabilistic learning style combinations: a qualitative comparison between two innovative stochastic approaches". Journal of The Brazilian Computer Society. Volume 19, Issue 1, pp 43-58, 2013.

[8] DORÇA, F. A. "Uma Abordagem Estocástica Baseada em Aprendizagem por Reforço para Modelagem Automática e Dinâmica de Estilos de Aprendizagem de Estudantes em Sistemas Adaptativos e Inteligentes para Educação a Distância". Tese de Doutorado. Programa de PósGraduação em Engenharia Elétrica. Universidade Federal de Uberlândia, 2012.

[9] ENTWISTLE, N. "Styles of learning and teaching”. Wiley Chichester, 1981. 
[10] FELDER, R. M.; BRENT, R. "Understanding Student Differences". Journal of Engineering Education, vol. 94, no. 1, 57-72, 2005.

[11] FELDER, R.; SILVERMAN, L. "Learning and teaching styles in engineering education". Engineering Education, 1988.

[12] FELDER, R.; SOLOMAN, B. "Index of Learning Style Questionnaire”, 2006. Disponível em: $<$ http://www4.ncsu.edu/unity/lockers/users/f/felde r/public/ILS page.html>. Acesso em: 10 out. 2012.

[13] FRANZONI, A. L.; ASSAR, S.; 2009, A. L.; ASSAR, S. "Student learning styles adaptation method based on teaching strategies and electronic media". Educational Technology \& Society, vol. 12, no. 4, 2009.

[14] GOMES, S. R.; GADELHA B. F., MENDONÇA, A.P.; AMORETTI, M. S. M. "Objetos de Aprendizagem Funcionais e as Limitações dos Metadados Atuais". Anais do XVI Simpósio Brasileiro de Informática na Educação - SBIE UFJF - 2005.

[15] GRAF S.; KINSHUK. "Advanced Adaptivity in Learning Management Systems by Considering Learning Styles". Proceedings of the 2009 IEEE/WIC/ACM International Joint Conference on Web Intelligence and Intelligent Agent Technology - Volume 03, IEEE Computer Society, 2009, 235-238.

[16] GRAF, S.; KINSHUK \& IVES, C. "A Flexible Mechanism for Providing Adaptivity Based on Learning styles". 10th IEEE International Conference on Advanced Learning Technologies, 30-34, 2010 .

[17] HONEY, P. and MUMFORD, A. "The manual of learning styles". Journal of Engineering Education, 94 (1), 57-72, 2005.

[18] IEEE LOM. "Draft standard for learning object metadata", 2002. Disponível em: $<$ http://ltsc.ieee.org/wg12/index.html $>$. Acesso em: 11 out. 2012.

[19] KOLB, D. "Experiential learning: Experience as the source of learning and development". Prentice-Hall Englewood Cliffs, NJ, 1984.

[20] LOPES, R. S. e FERNANDES, M. A.; "Adaptative Instructional Planning Using Workflow and Genetic Algorithms". In Computer and Infor- mation Science, 2009. ICIS 2009. Eighth IEEE/ACIS International Conference on, pages 87-92. IEEE, 2009.

[21] MACEDO, L. N. A. "Avaliação de um Objeto de Aprendizagem com Base nas Teorias Cognitivas". XIII Workshop em Informática Educativa, 330$338,2007$.

[22] MILOSEVIC, D. \& BRKOVIC, M. "Adaptive Learning by Using SCOs Metadata". Interdisciplinary Journal of Knowledge and Learning Objects, 2007, 3, 163-174.

[23] MODEL, O.; EL-KHOULY, M.; EL-SEOUD, S. "On line student model". International Journal of Computing \& Information Sciences, vol. 4, no. 1, 2006.

[24] NAT, M.; WALKER, S.; BACON, L.; DASTBAZ, M. "Designing personalization in LAMS". University of Greenwich; European LAMS \& Learning Design Conference, 120-127, 2010 .

[25] PAREDES, P.; RODRIGUES, P. "Considering Sensing-Intuitive Dimension to Exposition- Exemplification in Adaptive Sequencing”. Adaptive Hypermedia and Adaptive Web-Based Systems, Springer Berlin Heidelberg, 2002, 2347, 556-559.

[26] PASK, G. "Styles and strategies of learning". British journal of educational psychology, Volume 46, 128-148, 1976.

[27] ROCHA, F. L.; de MORAES, H. N.; FABRI, L. B. W.; de J. OLIVEIRA, T.; COSTA, A. C. R.; NETTO, C. M.; da COSTA, H. R. \& MORAIS, R. C. R. "Repositórios de objetos de aprendizagem - um estudo exploratório". Anais do XXII SBIE XVII WIE, 2011, 304-312.

[28] ROMERO, C.; VENTURA, S.; DELGADO, J. A. \& Bra, P. D. "Personalized Links Recommendation Based on Data Mining in Adaptive Educational Hypermedia Systems". EC-TEL'07 Proceedings of the Second European conference on Technology Enhanced Learning: creating new learning experiences on a global scale. Pages 292306. Springer-Verlag Berlin, Heidelberg, 2007

[29] SANGINETO, E., CAPUANO, N., GAETA, M., e MICARELLI, A. "Adaptive course generation through learning styles representation". Universal Access in the Information Society, 7(1):1-23, 2008. 
[30] SILVA, G. T. \& ROSATELLI, M. C. "Adaptation in educational hypermedia based on the classification of the user profile". Proceedings of the 8th international conference on Intelligent Tutoring Systems, Springer-Verlag, 2006, 268-277.

[31] TERRY, R. E., HARB, J. N., HURT, P., e WILLIAMSON, K. "Teaching through the cycle: application of learning style theory to engineering education at Brigham Young University". Brigham Young University Press, 1995.

[32] VAHLDICK, A.; RAABE, A. L. A. "Adaptação de Conteúdo SCORM em Ambientes Inteligentes de Aprendizagem". XIX Simpósio Brasileiro de Informática na Educação (SBIE), 2008.

[33] W3C OWL. "Web Ontology Language (OWL)", 2010. Disponível em: <http://www.w3 .org/2004/OWL/>. Acesso em: 17 out. 2012.

[34] W3C RDF. "Resource Description Framework (RDF)". 2010. Disponível em: <http://www. w3.org/RDF/>. Acesso em: 17 out. 2012.

[35] W3C XML. "Extensible Markup Language (XML)". 2010. Disponível em: <http://www.w3. org/XML/>. Acesso em: 17 out. 2012.

http://www.br-ie.org/pub/index.php/rbie
[36] W3C XQUERY. "XQuery 1.0: An XML Query Language". 2010. Disponível em: <http:// www.w3.org/TR/xquery/>. Acesso em: 17 out. 2012.

[37] WARPECHOWSKI, M. \& de OLIVEIRA, J. P. M. "Obtenção de Metadados de Objetos de Aprendizagem no AdaptWeb". Instituto de Informática - Universidade Federal do Rio Grande do Sul (UFRGS), 2001.

[38] WILEY, D. A., "Learning object design and sequencing theory". Tese de doutorado, Brigham Young University, Department of Instructional Psychology and Technology, 2000.

[39] ZAINA, L.A.M., BRESSAN, G. "Learning objects retrieval from contextual analysis of user preferences to enhance e-learning personalization". In: Proceedings of IADIS International Conference WWW/Internet, 2009.

[40] ZAINE, L.; BRESSAN, A.M.; CARDIERI, R. J.; e-LORS "Uma abordagem para recomendação de objetos de aprendizagem". Revista Brasileira de Informática na Educação. v. 20, p. 4-16, 2012. 